\title{
COMPORTAMIENTO DE TRES CULTIVARES DE CAFÉ (CATIMOR, COLOMBIA Y COSTA RICA 95) EN EL VALLE DE EL PERENÉ, JUNÍN, PERÚ
}

\section{BEHAVIOR OF THREE CULTIVARS OF COFFEE (CATIMOR, COLOMBIA AND COSTA RICA 95) IN THE VALLEY OF EL PERENÉ, JUNÍN, PERÚ}

\author{
Alberto Julca-Otiniano ${ }^{1 *}$, Gerson Alarcón-Águila ${ }^{1}$, Leonel Alvarado-Huamán ${ }^{1}$, \\ Ricardo Borjas-Ventura ${ }^{1}$, y Viviana Castro-Cepero ${ }^{2}$ \\ ${ }^{1}$ Dpto. Fitotecnia, Universidad Nacional Agraria La Molina (UNALM), Av. La Molina s/n, La Molina, \\ Lima. Perú. \\ ${ }^{2}$ Dpto. Biología, Universidad Nacional Agraria La Molina (UNALM), Av. La Molina s/n, La Molina, \\ Lima. Perú. \\ * Autor para correspondencia: ajo@lamolina.edu.pe
}

\section{RESUMEN}

El objetivo de este estudio fue evaluar el comportamiento de tres cultivares de café (Catimor, Colombia y Costa Rica 95) en el Valle de El Perené, Junín, Perú. El experimento se realizó en la Finca "Alto Florida", ubicada a $1250 \mathrm{msnm}$; para las evaluaciones se seleccionaron y marcaron $\mathbf{5 0}$ plantas de cada cultivar. El estudio no tuvo un diseño estadístico por lo que para el análisis estadístico se trabajó como si fuera un Diseño Completamente al Azar (DCA), donde los tres cultivares fueron considerados como tratamientos ( 1 cultivar $=1$ tratamiento) y las 50 plantas como repeticiones ( 1 planta $=1$ repetición). Se encontró que los tres cultivares estudiados tienen características fenotípicas muy similares. Pueden considerarse resistentes a la "roya del café" (Hemileia vastatrix) pero susceptibles a otros problemas fitosanitarios importantes como Hypothenemus hampei, Perileucoptera coffeella, Cercospora coffeicola y Mycena citricolor. Son de alto rendimiento en campo (mayor a 0,18 $\mathrm{t} \mathrm{ha}^{-1} \mathrm{de}^{-}$ café pergamino) y de buena calidad física (mayor a $72 \%$ de café exportable). También tienen buena calidad organoléptica (con 79,9 a 82,1 puntos) que las calificaría como café especial.

Palabras clave: Crecimiento, calidad física, calidad organoléptica, rendimiento, Hemileia

\section{ABSTRACT}

The objective of this study was to evaluate the behavior of three coffee cultivars (Catimor, Colombia and Costa Rica 95) in the Valley of El Perené Junín, Perú. The experiment was carried out in the "Alto Florida" farm, which is located at 1250 m.a.s.l. Fifty plants of each cultivar were selected and marked for the evaluations. A completely randomized design was used with 3 treatments (coffee cultivars) and 50 replicates ( 1 plant $=1$ replicate). The results indicate that the three cultivars studied have similar phenotypic characteristics. They can be considered resistant to "coffee rust" (Hemileia vastatrix), but susceptible to other important phytosanitary problems, such as Hypothenemus hampei, Perileucoptera coffeella, Cercospora coffeicola and Mycena citricolor. They present high yield (greater than $0.18 \mathrm{t} \mathrm{ha}^{-1}$ of parchment coffee) and good physical quality (greater than $72 \%$ of exportable coffee). They also have good organoleptic quality (with 79.9 to 82.1 points), which allows them to be described as a special coffee.

Key words: Growth, physical quality, organoleptic quality, yield, Hemileia

Recibido: 02 mayo 2018. Aceptado: 07 agosto 2018 


\section{INTRODUCCIÓN}

El café es el principal producto de agroexportación del Perú, siendo el octavo país productor de café en el mundo, pues cuenta con más de 420 mil hectáreas cultivadas y es el sustento de 223 mil familias (Ministerio de Agricultura y Riego - MINAGRI, 2018). Según el MINAGRI (2015), en el 2014 se tuvo una producción de 209 mil toneladas.

A pesar de la importancia económica del café, aun se tienen problemas muy serios que afectan la producción, como la "roya del café" (Hemileia vastatrix). En el 2013, el área afectada en el Perú, fue de $290 \mathrm{mil}$ ha con niveles de incidencia entre 30 a 60\% (MINAGRI, 2014) por lo que es necesario desarrollar experiencias para el diseño adecuado de programas de manejo integrado de esta enfermedad, en el que el uso de cultivares resistentes debería ser la piedra angular de esos programas (Julca et al., 2013).

El desarrollo de cultivares resistentes a la "roya del café" ha sido posible gracias a que existen fuentes naturales de resistencia, especialmente en el Híbrido de Timor ( $\mathrm{H}$ de $\mathrm{T})$. Por ejemplo, en América Central a través del PROMECAFE, se seleccionaron localmente diferentes cultivares resistentes a partir de genotipos híbridos llamados Catimor (Caturra $\times \mathrm{H}$ de $\mathrm{T}$ ), originarios de Portugal o Brasil. En Costa Rica todas las selecciones locales de Catimor (Catrenic, IH café 90 y Costa Rica 95) fueron realizadas con criterios de productividad y de resistencia a la raza II de la roya. Lamentablemente sus deficiencias en materia de calidad han hecho que su expansión no haya sido la esperada, y más del $90 \%$ del área sembrada con café en Centroamérica es todavía con cultivares susceptibles (López, 2010). Sin embargo, existen referencias que señalan que la calidad de los Catimores no difiere de los cultivares más tradicionales. Así tenemos que en Colombia no se encontraron diferencias estadísticas significativas en la calidad de la bebida al comparar el cv. Colombia con Típica, Caturra y Borbón (Alvarado y Puerta, 2002). Tampoco entre Castillo, Típica, Caturra, Borbón y Colombia (Alvarado et al., 2005). En México no se han reportado diferencias estadísticas significativas entre Oro Azteca, Colombia y Típica (Tres Cruces) para el aroma, la acidez, el sabor y el cuerpo de la taza (López-García et al., 2016). Quizá por ello, desde el año 2012, y luego del ataque de la roya en México, se viene renovando los cafetales con nuevos cultivares, entre los que están los Catimores (Juárez-Bravo et al., 2018).

En algunos países productores de café se tienen estudios referentes al comportamiento de cultivares evaluando no solo su grado de resistencia a $H$. vastatrix, sino también su crecimiento y desarrollo (Manchego et al., 1999; Alvarado et al., 2005). Estos estudios ayudan en la adecuada elección de cultivares con la finalidad de aumentar la productividad y calidad de la cosecha, y por lo tanto un mayor ingreso para los agricultores. En el Perú no existe una institución encargada de investigar la adaptación de nuevos cultivares de café a las condiciones locales y los estudios de comportamiento de cultivares frente a la "roya del café" son muy escasos. Por ello la necesidad de conservar, evaluar y recomendar el uso correcto del material genético, como ocurre en los países cafetaleros líderes que cuentan con instituciones dedicadas a trabajar exclusivamente en el desarrollo tecnológico de este cultivo, y donde el manejo de los recursos fitogenéticos juega un rol protagónico (Julca et al., 2010a).

En Perú se manejan cultivares de café como son Catimor, Colombia y Costa Rica 95 (Julca et al., 2010a) consideradas resistentes a la "roya del café" y su uso se viene extendiendo poco a poco; si bien estos cultivares se consideran resistentes a esta enfermedad, son susceptibles a otras plagas y enfermedades y son considerados de baja calidad organoléptica. Para documentar las características que se les atribuye, este ensayo tuvo como objetivo conocer el comportamiento de tres cultivares de café (Catimor, Colombia y Costa Rica 95) en el Valle de El Perené, Junín, Perú.

\section{MATERIALES Y MÉTODOS}

El experimento se realizó en la Finca "Alto Florida", localizada en el Valle de El Perené, provincia de Chanchamayo, región Junín, Perú. Está ubicada a $1250 \mathrm{msnm}$, a $10^{\circ} 54^{\prime} 11^{\prime \prime} \mathrm{S}$ y $75^{\circ} 15^{\prime} 1$ " $\mathrm{O}$ y tiene instaladas parcelas de los cultivares Catimor, Colombia y Costa Rica 95, todas de cuatro años de edad, con una densidad de 5000 pl ha $^{-1}(2 \times 1 \mathrm{~m})$ y bajo sombra de Inga sp. y Pinus sp. Es importante mencionar que en el país no existe semilla certificada de café, pero la presencia de estos tres cultivares ha sido confirmada en la zona de estudio en investigaciones realizadas anteriormente, y actualmente forman parte del banco de germoplasma de la Universidad Nacional Agraria La Molina, en San Ramón, Chanchamayo (Blas et al., 2011).

La fórmula de fertilización $\left(\mathrm{kg} \mathrm{ha}^{-1}\right)$ fue de $160 \mathrm{~N}-56 \mathrm{P}_{2} \mathrm{O}_{5}-160 \mathrm{~K}_{2} \mathrm{O}-10 \mathrm{~B}$ de aplicación fraccionada, la primera al inicio de campaña y la segunda antes de la floración.

Para las evaluaciones se seleccionaron y marcaron 50 plantas de cada cultivar, y las variables estudiadas correspondieron a la lista de descriptores de café publicado por el International 
Resources Institute - IPGRI (1996), a la que se le adicionó otras variables como la incidencia de plagas y enfermedades. Se evaluó: forma de la planta, ramificación, forma de la estípula, forma de la hoja, forma del ápice, color de la hoja madura, color de brote, color del pecíolo, color del fruto, forma del fruto, altura de planta, número de ramas, número de hojas, número de nudos, longitud de ramas, longitud de entrenudos, infestación de la "broca" (Hypothenemus hampei), infestación del "minador de hoja" (Perileucoptera coffeella), incidencia de "ojo de gallo" (Mycena citricolor), incidencia de la "cercosporiosis" (Cercospora coffeicola), incidencia de la "roya" (Hemileia vastatrix), peso de 100 frutos maduros, peso de café cerezo, peso de café pergamino seco, y relación café cerezo/café pergamino seco. La incidencia de las plagas y enfermedades fue medida usando la fórmula: (NOI/NOT) x100, donde NOI: Número de órganos afectados y NOT: Número de órganos totales.

La cosecha fue selectiva, es decir solo se cosecharon los frutos maduros, y luego se hizo el despulpado, fermentado, lavado y secado. Para evaluar la calidad se tomaron muestras con un peso mínimo de 300 gramos de café pergamino seco de cada unidad experimental. Estas se llevaron al Laboratorio de la Municipalidad de Villa Rica donde se evaluó la calidad física y organoléptica.

El estudio no tuvo un diseño estadístico por lo que para el análisis estadístico se trabajó como si fuera un Diseño Completamente al Azar (DCA), donde los tres cultivares fueron considerados como tratamientos ( 1 cultivar $=1$ tratamiento) y las 50 plantas como repeticiones ( 1 planta $=1$ repetición). Para el caso de plagas y enfermedades, los datos fueron previamente transformados con la ecuación $X^{\prime}=(\operatorname{arcsen} \sqrt{ } x)$.

\section{RESULTADOS Y DISCUSIÓN}

Los tres cultivares estudiados (Catimor, Colombia y Costa Rica 95) son resultado del cruce de Caturra x Híbrido de Timor (Julca et al., 2013). Por ello las características fenotípicas de estos tres cultivares son similares, con excepción del color del brote (hoja joven o "cogollo") que en Catimor era de color bronce más claro, lo que le da una tonalidad rojiza (Tabla 1). Esto es importante de señalar porque para algunos autores el color del brote es una característica que puede ser usada para diferenciar los cultivares de café (Calle, 2009). Sin embargo, también se ha reportado brotes de color verde y bronce en diferentes linajes de Catimor (Severino et al., 2001). Blas et al. (2011) señalan que el color de las hojas jóvenes está gobernado por dos genes $(\mathrm{Br}=$ bronceado $\mathrm{y} b r=$ verde) con dominancia incompleta y se encuentran tres genotipos y fenotipos $(\mathrm{BrBr}=$ bronce oscuro, $\mathrm{Brbr}=$ bronce claro, $\mathrm{y}$ brbr $=$ verde) .

\section{Crecimiento de la planta.}

El estudio del crecimiento es importante ya que tiene influencia en la producción de los cultivos (Carvalho et al., 2010). En este ensayo se encontró diferencias en altura de planta ( $p$ $\leq 0,05)$ ya que Costa Rica 95 tuvo una altura de 204,9 cm, seguido de Colombia y Catimor con 177,76 y $140,90 \mathrm{~cm}$, respectivamente (Fig. 1A). Colombia es considerada un cultivar de porte bajo (Anzueto, 2014) y Severino et al. (2002) señalan que la altura de planta está relacionada con el cultivar y tiene una alta heredabilidad (96\%). Para el número de ramas, Catimor $(34,08)$ y Colombia $(39,4)$ no mostraron diferencias estadísticas significativas entre sí, pero fueron menores $(p \leq 0,05)$ comparadas con Costa Rica $95(55,08)$ tal como se muestra en la Fig. 1B. Esta mayor cantidad de ramas primarias encontradas en Costa Rica 95 se explica por una mayor altura de planta. Los valores obtenidos en Catimor y Colombia, se encuentran en el rango reportado por Manchego et al. (1999).

Los nudos de las ramas son importantes porque en ellos se originan las flores cuando las condiciones climáticas son óptimas (Arcila et al., 2007). De forma general se observó que tanto en Catimor como en Costa Rica 95 hubo una disminución en el número de nudos, desde el tercio inferior hasta el tercio superior. Si bien la cantidad de nudos en el tercio inferior y medio no fueron estadísticamente diferentes en cada uno de estos cultivares, si fueron mayores ( $p \leq 0,05$ ) a la del tercio superior. Asimismo, al comparar el número de nudos del tercio inferior y superior entre cada uno de los cultivares, se encontró que Costa Rica 95 tuvo mayor cantidad de nudos ( $\mathrm{p} \leq$ 0,05 ) que los cultivares Catimor y Colombia (Fig. 1C). Pero en el tercio medio, el número de nudos de Costa Rica $95(15,92)$ y Colombia $(14,28)$, fueron estadísticamente similares $(\mathrm{p} \leq 0,05)$ y superiores al de Catimor $(\mathrm{p} \leq 0,05)$. Diferencias en el número de nudos entre cultivares, también se han reportado para el caso del café Robusta (Santistevan, 2013).

La longitud de entrenudos en cada uno de los cultivares fue variable (Fig. 1D). Colombia tuvo estadísticamente la mayor longitud ( $\mathrm{p} \leq$ $0,05)$ comparado con los otros dos cultivares, alcanzando los valores de $4,70 \mathrm{~cm}, 4,36 \mathrm{~cm}$ y $3,54 \mathrm{~cm}$ para el tercio inferior, medio y superior, respectivamente. En los tres cultivares, la longitud de ramas (Fig. 1E) disminuyó desde el tercio inferior hasta el tercio superior, aunque la 
Tabla 1. Características fenotípicas de los tres cultivares de café evaluados en el Valle de El Perené, Junín, Perú.

Table 1. Fenotypic characteristics of three coffee cultivars evaluated in the Valle de Parené, Junín, Peru.

\begin{tabular}{llll}
\hline Variable & Catimor & Colombia & Costa Rica 95 \\
\hline Forma de la planta & Cónico & Cónico & Cónico \\
Ramificación primaria & Mucha & Mucha & Mucha \\
Ángulo de inserción & Erguido & Erguido & Erguido \\
Forma de estípula & Triangular & Triangular & Triangular \\
Forma de hoja & Elíptica & Elíptica & Elíptica \\
Forma de ápice & Apiculada & Apiculada & Apiculada \\
Color de hoja & Verde oscuro & Verde oscuro & Verde oscuro \\
Color del brote & Bronce claro & Bronce & Bronce \\
Color de peciolo & Verde & Verde & Verde \\
Color del fruto & Rojo & Rojo & Rojo \\
Forma del fruto & Oblongo & Oblongo & Oblongo \\
\hline
\end{tabular}

longitud en el tercio inferior y del tercio medio no fueron estadísticamente diferentes. Al comparar cultivares, Catimor tuvo estadísticamente el menor valor $(p \leq 0,05)$ en el tercio inferior $(42,10$ $\mathrm{cm})$, medio $(42,46 \mathrm{~cm})$ y superior $(24,64 \mathrm{~cm})$. Es decir, Catimor es un cultivar con un menor número de nudos y una menor longitud de entrenudos comparado con Colombia y Costa Rica 95. En general, la mayor longitud de ramas en la parte inferior y media confiere a las plantas de café la forma piramidal, tal como reporta Favarin et al. (2002).

Las hojas están relacionadas con el rendimiento potencial del café (Favarin et al., 2002) porque en estas ocurren los procesos fisiológicos (fotosíntesis, respiración y transpiración) en los que se basa el crecimiento y desarrollo de la planta (Arcila et al., 2007). En este ensayo los cultivares Catimor y Colombia tuvieron mayor cantidad de hojas en el tercio medio comparado con Costa Rica 95. Para Catimor no se encontraron diferencias ( $p$ $\leq 0,05$ ) entre los tres tercios evaluados, mientras que en el cultivar Colombia el número de hojas del tercio medio fue mayor $(\mathrm{p} \leq 0,05)$ al superior $\mathrm{e}$ inferior. En Costa Rica 95, el tercio superior tuvo mayor cantidad de hojas $(p \leq 0,05)$ que el tercio medio e inferior (Fig. 1F). En general, el número de hojas/rama fue similar para los tres cultivares: $48,02,49,68$ y 45,60 para Catimor, Colombia y Costa Rica 95, respectivamente.

\section{Incidencia de plagas y enfermedades}

Para Bustillo (2006), la "broca" (H. hampei) es la plaga más importante del café a nivel mundial y, según Montoya (1999) existe una relación entre los daños en los granos de café y la menor calidad de la bebida. De forma general, se tuvo un mayor nivel de infestación en el cultivar Catimor (0,54\%), sin embargo, la diferencia no fue significativa respecto a los otros dos cultivares que tuvieron valores similares $(0,26 \%)$ tal como se muestra en la Fig. 2A. La "broca" fue aumentando desde el tercio inferior hasta el tercio superior, pero los niveles de infestación detectados en el ensayo fueron menores al umbral de acción (5\%) señalado por Bustillo (2006). En Perú, Acacio y Gil (2012) reportaron que el nivel de infestación de esta plaga en Catimor es alto y varía de 8,36 a 23,12\%. Un nivel alto de infestación en Catimor (35,3\%) también ha sido reportado cuando se lo comparó con los cultivares Típica $(24,7 \%)$ y Caturra Roja $(13 \%)$ en un estudio realizado en Chanchamayo en la selva central del Perú (Julca et al., 2007).

El "minador de la hoja" (Perileucoptera coffeella) es otra de las plagas importantes del café, ya que el daño es causado por las larvas que se alimentan de hojas, que en altas infestaciones presenta una incidencia que puede llegar a $80 \%$, lo que se refleja en una menor producción (Constantino et al., 2011). En este estudio (Fig. 2B) el nivel de infestación fue diferente para los tres cultivares y el valor más alto correspondió a Costa Rica 95 (16,54\%), seguido de Colombia (14,70\%) y Catimor (9,52\%), valores bajo el umbral de acción del 20\% según lo reportado por Constantino et al. (2011). En otros cultivares de café como Conilon, se encontró un nivel de infestación de esta plaga del $77 \%$ en el tercio superior de la planta (Costa et al., 2001).

El "ojo de gallo" (Mycena citricolor) no mostró diferencias entre las partes de la planta evaluadas dentro de cada cultivar. Sin embargo, al comparar la incidencia en el tercio medio de los tres cultivares, se encontró que la enfermedad fue mayor $(p \leq 0,05)$ en Costa Rica 95 comparada con 

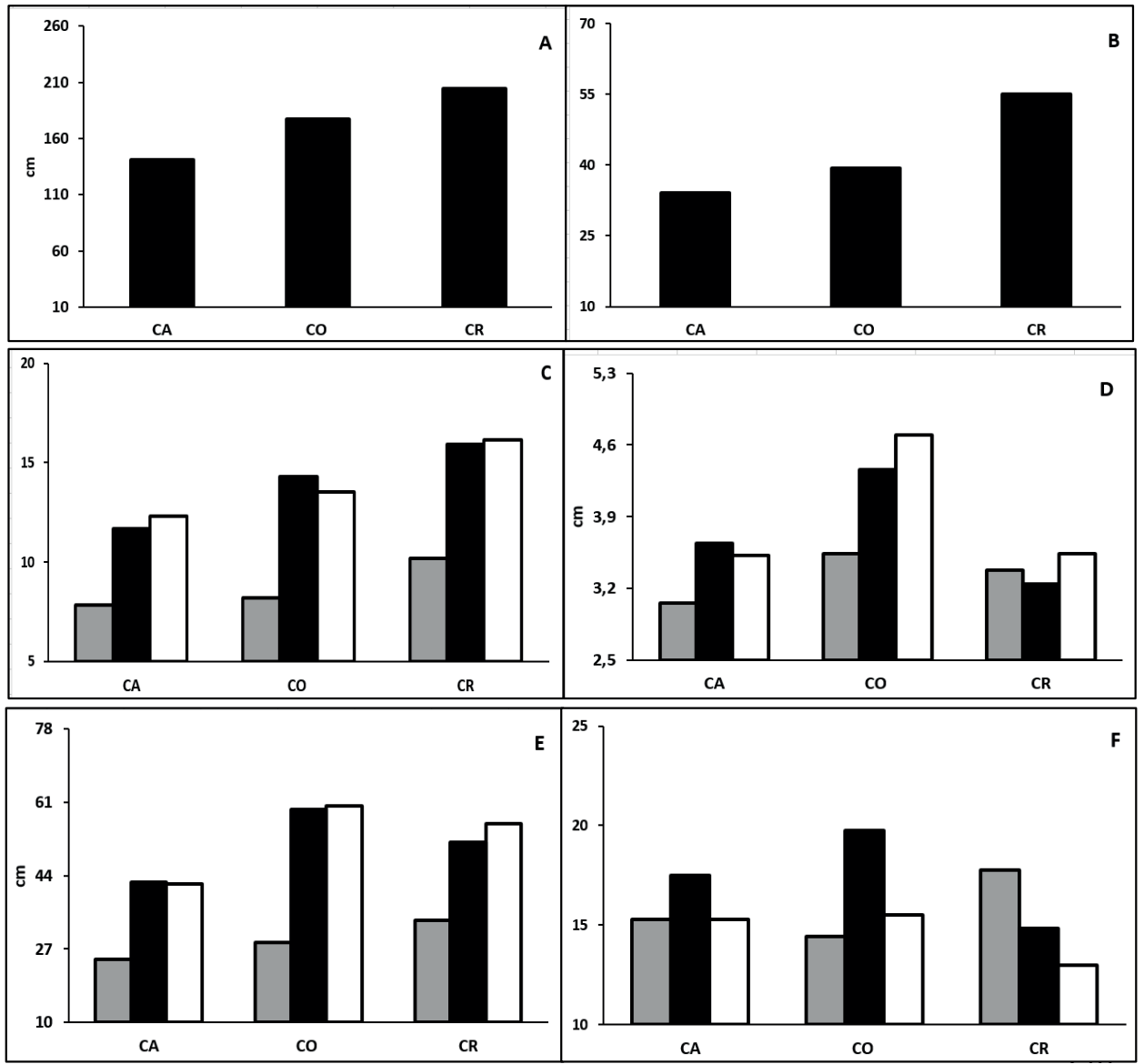

Fig. 1. Variables de crecimiento en los tres cultivares de café evaluados en el experimento. Altura de planta (A), número de ramas (B), número de nudos (C), longitud de entrenudos (D), longitud de ramas (E) y número de hojas (F) en los cultivares Catimor (CA), Colombia (CO) y Costa Rica 95 (CR). En C, D, E, F: Barra gris (tercio superior), barra negra (tercio medio) y barra blanca (tercio inferior).

Fig. 1. Growth variables in the three coffee cultivars evaluated in the experiment. Plant height (A), number of branches (B), number of nodes (C), Length of internodes (D), Length of branches (E) and number of leaves (F) in the cultivars Catimor (CA), Colombia (CO) and Costa Rica 95 (CR). In C, D, E, F: Grey bar (top third), black bar (medium third) and white bar (lower third).

Catimor (Fig. 2C). Costa Rica 95 es considerado un cultivar altamente susceptible a esta enfermedad (Aguilar, 1995), y en este estudio presenta una incidencia casi similar a Colombia (12,56 y $12,66 \%$, respectivamente) valores mucho más altos que Catimor (5,86\%). Sin embargo, en este último se han reportado incidencias más altas, por ejemplo, Julca et al. (2010b) reportaron una incidencia de $44,3 \%$ en el cultivar Catimor en un estudio realizado en Villa Rica, en la selva central peruana, por lo que los tres cultivares podrían ser considerados como susceptibles a esta enfermedad.

Cercospora coffeicola puede causar pérdidas en el cultivo de café entre 15 a 30\% (Pozza et al., 2001). En este estudio no hubo diferencias estadísticas de incidencia entre los tercios inferior, medio y superior de cada cultivar $(\mathrm{p} \leq$ 0,05). Pero en general, en los cultivares Catimor y Colombia, la incidencia fue mayor en el tercio superior, pero no en Costa Rica 95 (Fig. 2D). Julca et al. (2010b) encontraron que en el cultivar Catimor, la incidencia fue mayor en el tercio superior que es la parte de la planta más expuesta a la luz. Los mismos autores señalaron que, en trabajos realizados a nivel de microscopio, se encontró que en hojas de café cultivado bajo sombra la penetración del hongo por los estomas fue reducida y que se desarrollaron pocas lesiones en las hojas. Pero el hongo no solamente necesita nutrientes y buenas condiciones de temperatura, sino también luz para la producción 
de la cercosporina, una toxina relacionada con el proceso de patogénesis (Julca et al., 2010b). La incidencia diferente de las enfermedades en cada tercio de la planta sugeriría que los patógenos no solamente encuentran condiciones ambientales y nutritivas favorables en partes específicas del cafeto, sino también que existiría una competencia entre estos por colonizar el follaje de la planta, como se ha reportado entre M. citricolor y C. coffeicola en el cultivar Catimor (Julca et al., 2010b). Por otro lado, si se suma la incidencia de $C$. coffeicola en los tres tercios de la planta, se tiene que Costa Rica 95 tuvo la mayor incidencia $(20,34 \%)$, seguido de Colombia $(16,94 \%)$ y Catimor $(8,16 \%)$, valores que están por encima del $5 \%$ señalado como el umbral de acción por Montes et al. (2012) y, por lo tanto, los tres cultivares podrían ser considerados como susceptibles a esta enfermedad.

La "roya del café" (Hemileia vastatrix) es considerada la enfermedad más devastadora del cultivo de café (Agrios, 1997, citado por
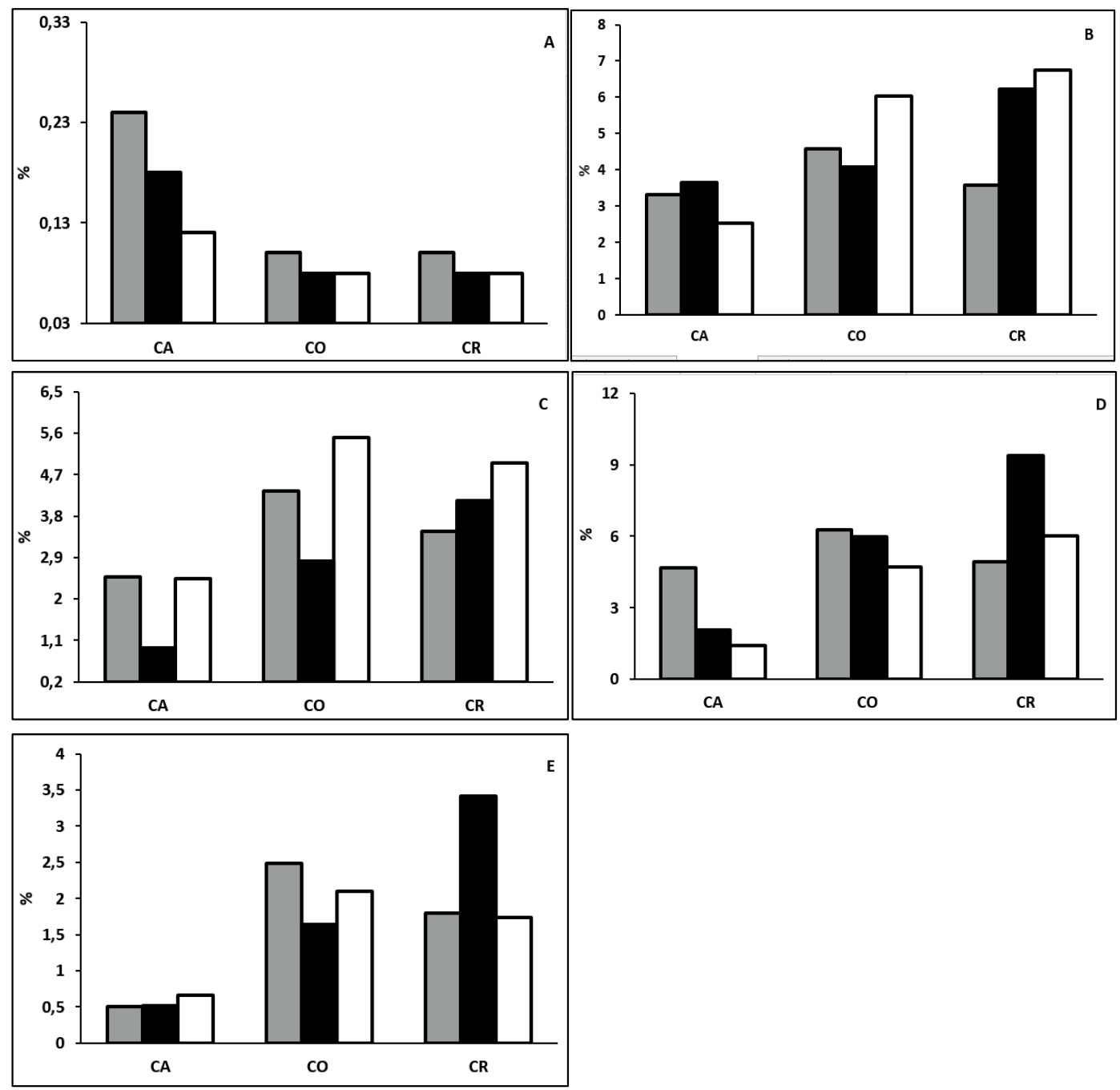

Fig. 2. Variables relacionadas a la sanidad del cultivo de café evaluadas en este ensayo. Infestación de H. hampei (A), infestación de P. coffeella (B), incidencia de M. citricolor (C), incidencia de C. coffeicola (D), incidencia de H. vastatrix (E) en los cultivares Catimor (CA), Colombia (CO) y Costa Rica 95 (CR). Barra gris (tercio superior), barra negra (tercio medio) y barra blanca (tercio inferior).

Fig. 2. Variables related to the health of the coffee crop evaluated in this test. Infestation of H. hampei (A), infestation of $P$. coffeella (B), incidence of M. citricolor (C), incidence of C. coffeicola (D), incidence of $H$. vastatrix (E) in the cultivars Catimor (CA), Colombia (CO) and Costa Rica 95 (CR). Grey bar (top third), black bar (medium third) and white bar (lower third). 
Julca et al., 2010b). En este estudio la incidencia de la enfermedad en los tres cultivares fue baja pues el menor valor correspondió a Catimor $(1,68 \%)$, seguido de Colombia (6,22\%) y Costa Rica 95 (6,96\%), tal como se muestra en la Fig. 2E. Colombia es un cultivar compuesto y considerado resistente a esta enfermedad (Moreno y Alvarado, 2000). Catimor y Costa Rica 95 fueron seleccionados con criterios de productividad y resistencia a la raza II de la roya (López, 2010). Los tres cultivares son el resultado de cruzar Caturra x Híbrido de Timor y son considerados resistentes; pero resistencia no es sinónimo de inmunidad. Moreno y Alvarado (2000) señalaron que la resistencia se define como un estado de menos enfermedad o como la capacidad de un hospedero para limitar el crecimiento de un patógeno y es verdadera cuando es controlada por uno o más genes.

Capacidad productiva de la planta.

El número de frutos/rama fue mayor en el tercio medio de los cultivares Catimor y Colombia, pero en Costa Rica 95 el mayor número de frutos fue encontrado en la parte superior (Fig. 3A). Este último también tiene la mayor cantidad de frutos $(241,12)$ seguida de Catimor $(186,96)$ y
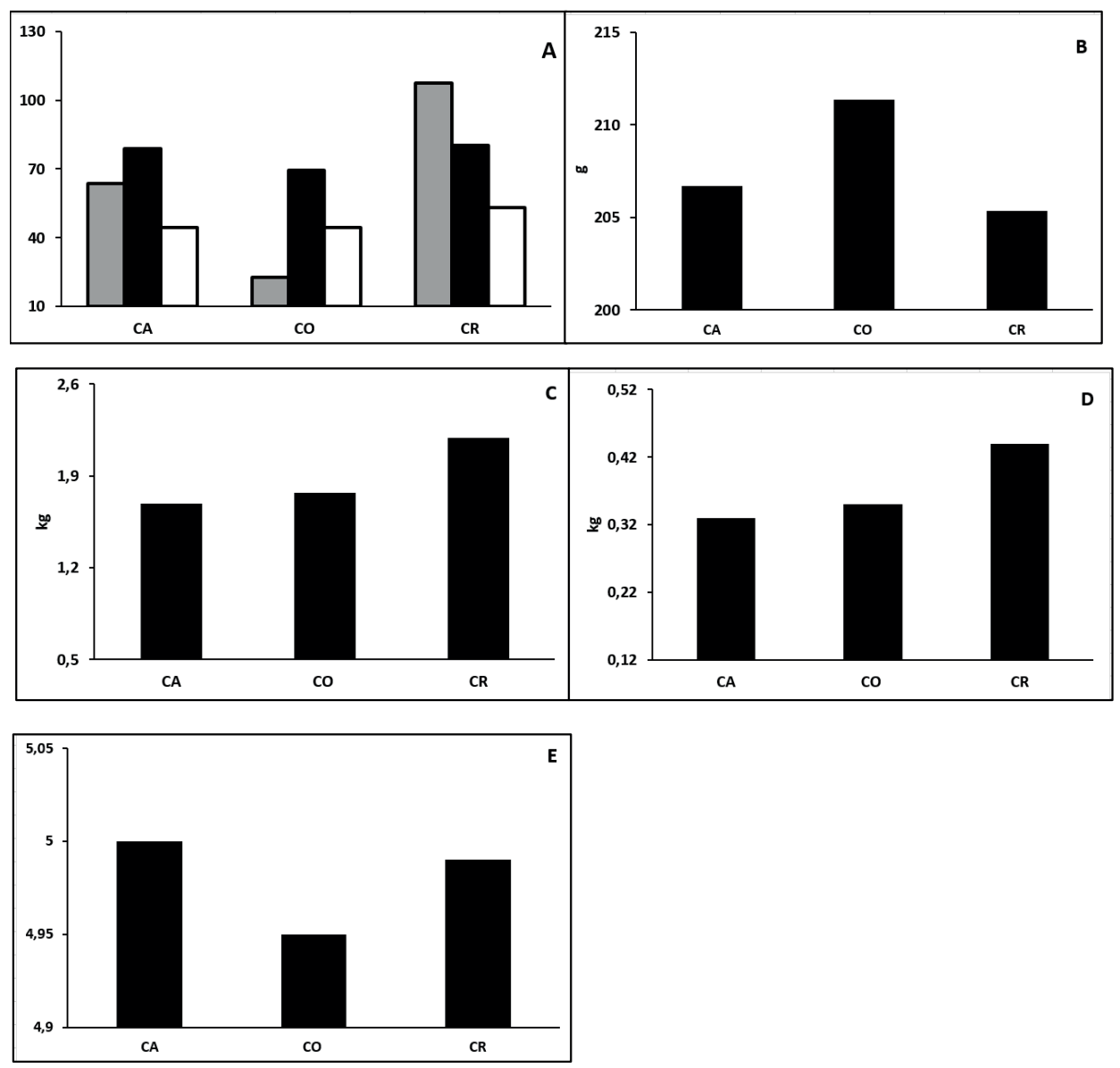

Fig. 3. Variables relacionadas a la producción del cultivo de café. Número de frutos/rama (A), peso de 100 granos de café cerezo (B), peso café cerezo/planta (C), peso café pergamino/planta (D), relación café cerezo/café pergamino (E) en los cultivares Catimor (CA), Colombia (CO) y Costa Rica 95 (CR). En A: Barra gris (tercio superior), barra negra (tercio medio) y barra blanca (tercio inferior).

Fig. 3. Variables related to the production of the coffee crop. Number of fruits / branch (A), weight of 100 grains of coffee cherry (B), weight coffee cherry/plant (C), weight coffee parchment/plant (D), relationship coffee cherry / coffee parchment (E) in the cultivars Catimor (CA), Colombia (CO) and Costa Rica 95 (CR). In A: Grey bar (top third), black bar (medium third) and white bar (lower third). 
Colombia $(136,70)$. El mayor número de frutos por rama en el tercio superior en Costa Rica 95 puede deberse a que este cultivar tiene la mayor cantidad de hojas en la parte superior, por tanto, mayor área fotosintética. Rao y Setly (1953) y Phillips (1970) citados por Da Matta et al. (2007), señalaron que el número de hojas es uno de los diversos factores que afectan la fructificación del café, pues la defoliación severa puede causar caída de los frutos más jóvenes; la caída de los frutos se produce principalmente en los primeros tres meses después de la floración y parece estar relacionado con el comienzo de la formación del endospermo, que se ha asociado parcialmente, con un bajo suministro de carbohidratos (Da Matta et al., 2007). Al cultivar Colombia le correspondió el mayor peso para 100 frutos o cerezos maduros $(p \leq 0,05)$, seguido de Catimor y Costa Rica 95 (Fig. 3B). Pero el mayor peso de café cerezo/planta correspondió al cultivar Costa Rica $95(2,19 \mathrm{~kg})$, mientras que el menor valor fue obtenido por Catimor $(1,69 \mathrm{~kg})$ tal como se muestra en la Fig. 3C. Anzueto (2013) reporta que una de las características de Costa Rica 95 es su alta productividad. Manchego et al. (1999) encontraron pesos de 2,225 a 3,089 $\mathrm{kg}$ de café cerezo en plantas de tres años de edad. Pero el mayor rendimiento de Costa Rica 95 es por una mayor cantidad de frutos/planta y no por el tamaño de fruto o cerezo pues es el cultivar Colombia el que tiene los frutos más grandes.

La producción de café pergamino/planta fue más alta en Costa Rica $95(0,44 \mathrm{~kg})$, valor que fue estadísticamente mayor $(\mathrm{p} \leq 0.05)$ con respecto a Colombia $(0,35 \mathrm{~kg})$ y Catimor $(0,33 \mathrm{~kg})$ que fueron estadísticamente similares (Fig. 3D), lo que contrasta con lo reportado por Contreras y Adriazola (2011) en Villa Rica, donde se encontró una producción de $0,58 \mathrm{~kg} /$ planta en el cultivar Colombia. Es importante señalar que los tres cultivares tuvieron una productividad mayor al promedio nacional estimado en $0,69 \mathrm{t} \mathrm{ha}^{-1}$, pues Costa Rica 95 tuvo un rendimiento de 2,20 $\mathrm{tha}^{-1}$ seguido de Colombia $\left(1,75 \mathrm{t} \mathrm{ha}^{-1}\right)$ y Catimor $(1,65$ $\left.\mathrm{t} \mathrm{ha}^{-1}\right)$, cálculos que fueron realizados con una densidad de plantación de 5000 plantas ha ${ }^{-1}$.

En la relación café cerezo/café pergamino (Fig. 3E), el menor valor correspondió al cultivar Colombia $(4,95)$ lo que es consistente con lo encontrado por Marín et al. (2003). Según los mismos autores, cuando los frutos fueron colectados en estados maduros y sobre maduros alcanzaron valores de conversión de 5,04 y 5,25, respectivamente. Los factores de conversión son importantes en la economía del café, pues los agricultores basan sus decisiones comerciales en esos factores (Montilla et al., 2008). En el Perú, esta información va tomando cada vez mayor importancia porque algunas organizaciones cafetaleras han construido plantas para centralizar el proceso de beneficio y mejorar la calidad del café, lo que implica un cambio importante para los agricultores que están acostumbrados a entregar a café pergamino y no café cerezo.

\section{Calidad física y organoléptica.}

La calidad del café es una característica ligada al sistema de producción y a los consumidores (ANACAFÉ, 2005). La calidad física o rendimiento de exportación (Fig. 4E) en Costa Rica 95 (76,75\%) fue estadísticamente mayor ( $\mathrm{p} \leq 0.05)$ que en Catimor $(73,62 \%)$ y en Colombia $(72,72 \%)$. En Perú se encontró que la calidad física variaba de una zona cafetalera a otra: en la selva central (Chanchamayo) fue de $74 \%$, mientras que en el sur (Quillabamba) fue de $68 \%$ (Julca et al., 2003). Posteriormente Julca et al. (2013) reportaron para la selva central del Perú, valores entre 75,4\% (San Ramón) y 80,3\% (Villa Rica) para el cultivar Caturra Roja. Según Fajardo y Sanz (2003) la disminución de la calidad física es causada por la baja selectividad en la cosecha y el tipo de beneficio del café. En el caso peruano, las diferencias en el rendimiento de exportación también son atribuidas a la falta de una infraestructura adecuada para el beneficio del café, un problema muy común entre los pequeños productores (Julca et al., 2003).

En cuanto a la calidad organoléptica, se tiene que el aroma es la primera cualidad que se percibe en el café, y depende de una gran cantidad de sustancias (Puerta, 2000a). En este estudio no se encontraron diferencias entre los cultivares estudiados en relación al aroma (Fig. 4A). Sin embargo, Puerta (2000b) encontró que el cultivar Colombia tuvo una alta puntuación cuando fue comparado con Típica y Bourbon. Para el sabor (Fig. 4B) el mayor puntaje fue obtenido por Colombia, seguido de Costa Rica 95 y de Catimor, con 7,59, 7,41 y 7,17, respectivamente ( $\mathrm{p} \leq 0,05)$. La acidez es una de las características más deseadas en el café arábica, aunque puede disminuir la calidad del café cuando se tiene malas prácticas de cosecha (Puerta, 2000a). En este ensayo la acidez fue mayor en Colombia (Fig. 4C), lo que coincide con lo reportado por Alvarado y Puerta (2002), que señalan que el cultivar Colombia se caracteriza por tener una alta acidez. En la evaluación del cuerpo del café (Fig. 4D), Colombia fue superior a los otros cultivares con 7,43 puntos $(\mathrm{p} \leq 0.05)$, siendo estos resultados similares a los reportados por Alvarado y Puerta (2002).

En general, el cultivar Colombia mostró mayor puntaje para calidad de taza $(82,05)$ seguido de Costa Rica $95(80,89)$ y de Catimor $(79,93)$ ( $\mathrm{p} \leq$ 

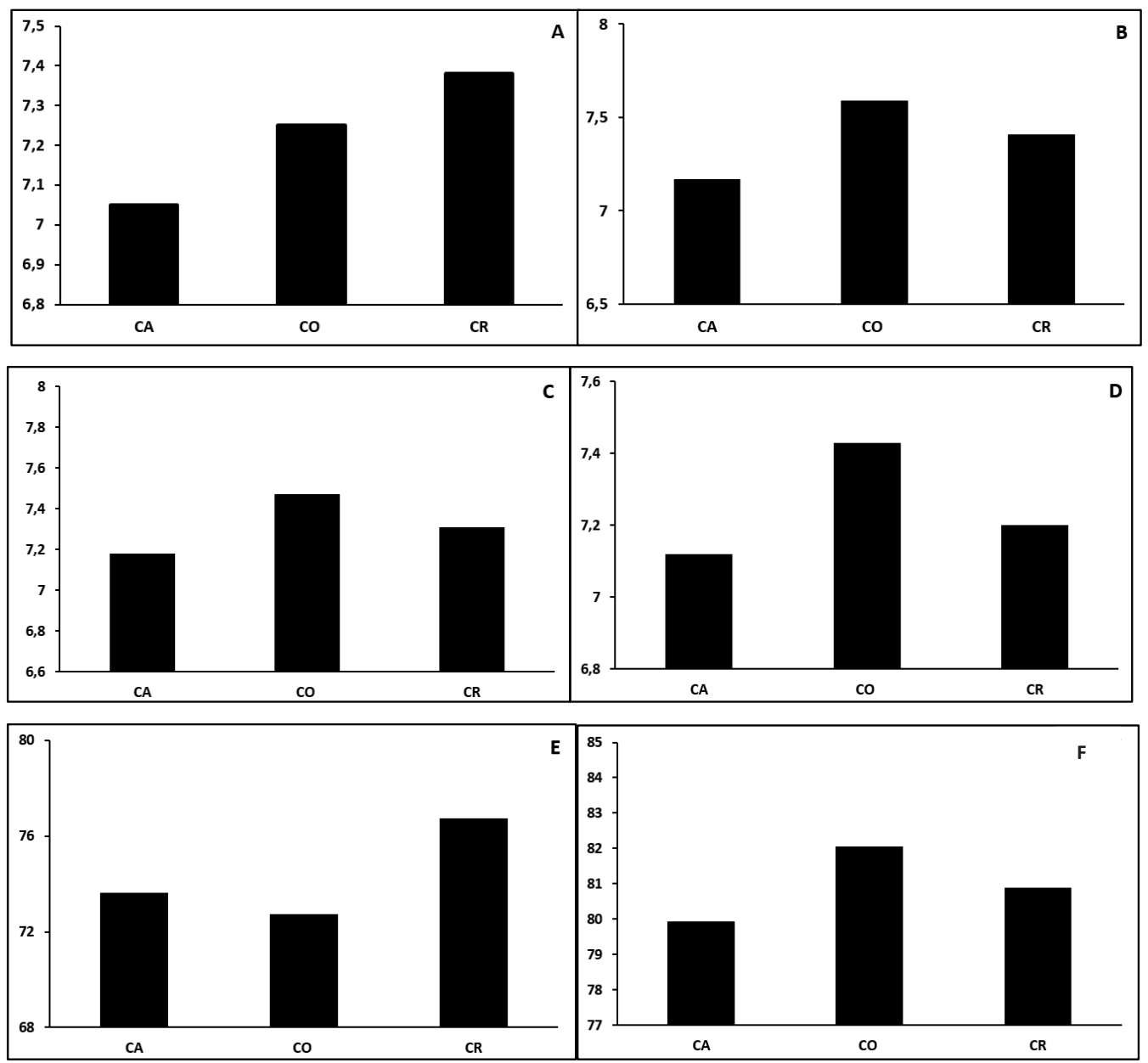

Fig. 4. Características de calidad. Aroma (A), sabor (B), acidez (C), cuerpo (D), calidad física (E), calidad organoléptica (F) en los cultivares Catimor (CA), Colombia (CO) y Costa Rica 95 (CR).

Fig. 4. Quality characteristics. Aroma (A), taste (B), acidity (C), body (D), physical quality (E), organoleptic quality (F) in the cultivars Catimor (CA), Colombia (CO) and Costa Rica 95 (CR).

0,05) como se muestra en la Fig. 4F. Si bien, existe una correlación entre la calidad física y la calidad sensorial de los granos de café (Puerta, 2000b) en este ensayo, el sabor, acidez y cuerpo fueron características que permitieron que el cultivar Colombia obtenga una puntuación superior. Mayor calidad de taza en el cultivar Colombia comparado con Costa Rica 95, fue reportado por Marín et al. (2003) en un estudio realizado en Colombia. Sin embargo, Da Matta et al. (2007) señala que la calidad de taza, además de los antecedentes genéticos, parece altamente dependiente de las condiciones climáticas y los procedimientos de recolección y post-cosecha, aunque queda por determinar cómo surge tal dependencia.

Los puntajes de calidad de taza alcanzados por los tres cultivares (Colombia $=82,05$; Costa Rica $95=80,89$; Catimor $=79,93)$ son relevantes, si se tiene en cuenta que un café de 80 puntos califica como especial, según la escala de la Specialty Coffee Association of America (SCAA). Julca et al. (2013) señalan que, en Perú, la baja calidad de taza atribuida al cultivar Catimor, es una apreciación subjetiva ya que, en ensayos realizados en la selva central, la calidad organoléptica no difiere mucho de la alcanzada por otros cultivares. Resultados similares han sido reportado en otros países productores de café, por ejemplo en Colombia, no se encontraron diferencias estadísticas significativas en la calidad de la bebida al comparar el cv. Colombia con Típica, Caturra y Borbón (Alvarado y Puerta, 2002). Tampoco entre Castillo, Típica, Caturra, Borbón y Colombia 
(Alvarado et al., 2005). En México, no se han encontrado diferencias estadísticas significativas entre Oro Azteca, Colombia y Típica (Tres Cruces) para el aroma, la acidez, el sabor y el cuerpo de la taza (López-García et al., 2016).

\section{CONCLUSIONES}

Los tres cultivares de café estudiados (Catimor, Colombia y Costa Rica 95) tienen características fenotípicas muy similares. Pueden considerarse resistentes a la "roya del café" pero susceptibles a otros problemas fitosanitarios importantes como H. hampei, P. cofeella, C. coffeicola y M. citricolor. Son de alto rendimiento en campo (mayor a $0,18 t$ ha $^{-1}$ de café pergamino) y de buena calidad física (mayor a $72 \%$ de café exportable). También tienen buena calidad organoléptica (con 79,9 a 82,1 puntos) que los calificaría como cafés especiales.

\section{LITERATURA CITADA}

Acacio, G., y J. Gil. 2012. Efecto del color de la trampa en la captura de la broca del café (Hypothenemus hampei Ferr) en tres localidades de Tingo María. Investigación y Amazonia 2(1-2):27-34.

Aguilar, G. 1995. Variedad Costa Rica 95. 30 p. Instituto del Café de Costa Rica. San José, Costa Rica. Convenio ICAFE-MAG. ISBN 977-55-014-X.

Alvarado, G., y G. Puerta. 2002. La variedad Colombia y sus características de calidad física y en taza. 3 p. Avances Técnicos 303. CENICAFÉ, Caldas, Colombia.

Alvarado, A., H. Posada, y H. Cortina. 2005. La variedad Castillo: Una variedad de café Coffea arabica L. con elevada productividad y amplia resistencia a enfermedades. Fitotecnia Colombiana 8:1-21.

Anzueto, F. 2013. Variedades de café resistentes la roya. Revista El Cafetal. ANACAFÉ. Disponible en https://www.anacafe.org/ glifos/index.php/Variedades_resistentes_a_ roya (Consulta 2 septiembre 2016).

Anzueto, F. 2014. Variedades de café. 9 p. Boletín Técnico. Asociación Nacional de Café de Guatemala (ANACAFÉ), Ciudad de Guatemala, Guatemala.

ANACAFÉ. 2005. La calidad del café y su importancia. Asociación Nacional de Café de Guatemala (ANACAFE). Disponible en https://www.anacafe.org/glifos/index. php?title=BeneficioHumedo_Calidad (Consulta 10 septiembre 2016).
Arcila, J., F. Farfán, A. Moreno, L. Salazar, y E. Hincapié. 2007. Sistemas de producción de café en Colombia. 309 p. 1a. ed. CENICAFÉ, Chinchiná, Colombia.

Blas, R., R. Cruz, S. Bello, R. Borjas, D. Talaverano, C. Echevarría, et al. 2011. Caracterización del germoplasma peruano de café. $57 \mathrm{p}$. UNALM-FDA-Café Perú-FINCyT. Editorial ESERGRAF, Lima, Perú.

Bustillo, A. 2006. Una revisión sobre la broca del café, Hypotenemus hampeii (Coleoptera: Curculionidae: Scolytinae) en Colombia. Rev. Colomb. Entomol. 32(2):101-116.

Calle, F. 2009. Calidad en taza y caracterización del color de las hojas jóvenes de 22 variedades de café. Tesis de Ingeniero Agrónomo. Escuela Agrícola Panamericana Zamorano, San Antonio de Oriente, Honduras.

Carvalho, A., A. Mendes, G. Carvalho, C. Botelho, F. Gonçalves, y A. Ferreira. 2010. Correlação entre crescimento em diferentes regiões de Minas Gerais. Pesqui. Agropecu. Bras. 45(3):269-275.

Constantino, L., J. Flórez, P. Benavides, y T. Bacca. 2011. Minador de las hojas del cafeto, una plaga potencial por efectos del cambio climático. 12 p. Avances Técnicos 409. CENICAFÉ, Caldas, Colombia.

Contreras, E., y J. Adriazola. 2011. Efecto de la pulpa de café y la fertilización química en el rendimiento y en la macrofauna edáfica del cultivo de café. Investigación y Amazonia 1(2):53-58.

Costa, J., P. Ribeiro, R. Silva, O. Trevisan, y J. Santos. 2001. Incidência do bicho-mineiro Leucoptera coffeella (Lepidoptera: Lyonetiidae) em café conilon no estado de Rondônia. p. 1956. En II Simposio de Pesquisa de Cafes do Brasil. 24-27 Setembro 2001. Espirito Santo, Brasil.

Da Matta, F., C. Ronchi, M. Maestri, and R. Barros. 2007. Ecophysiology of coffee growth and production. Braz. J. Plant. Physiol. 19(4):485510.

Fajardo, I., y J. Sanz. 2003. Evaluación de la calidad física del café en los procesos de beneficio húmedo tradicional e ecológico. CENICAFÉ 54(4):286-296.

Favarin, J., D. Neto, A. García, N. Nova, y M. Favarin. 2002. Equações para a estimativa do índice de área foliar do cafeeiro. Revista Agropecuaria Brasileira 37(6):769-773.

IPGRI. 1996. Descriptores de café. International Plant Genetic Resources Institute (IPGRI). Disponible en http://pdf.usaid.gov/pdf_ docs/Pnach860.pdf. (Consulta 25 septiembre 2016). 
Julca, A., R. Carhuallanqui, y R. Crespo. 2003. Rendimiento industrial del café para exportación en diferentes zonas cafetaleras del Perú. Café Cacao 4(2):3-7.

Julca, A., B. Guerrero, R. Adauto, L. Meneses, S. Bello, y R. Crespo. 2007. Infestación dela broca (Hypothenemus hampei) en tres variedades de Coffea arabica en Chanchamayo, selva central del Perú. 51 p. En Resúmenes del XLIX Convención Nacional de Entomología, Lambayeque, Perú.

Julca, A., R. Blas, R. Borjas, S. Bello, J. Anahui, D. Talaverano, et al. 2010a. Informe de colecta de germoplasma de café en el Perú. 38 p. UNALM-FDA-Café Perú-FINCyT. Lima, Perú.

Julca, A., R. Carhuallanqui, N. Julca, S. Bello, R. Crespo, C. Echevarría, et al. 2010b. Efecto de la sombra y la fertilización sobre las principales plagas del café var. Catimor en Villa Rica (Pasco, Perú). 23 p. UNALM-FDA, Lima, Perú.

Julca, A. C. Echevarría, Y. Ladera, R. Borjas, R. Cruz, S. Bello, et al. 2013. Una revisión sobre la roya del café (Hemileia vastatrix) algunas experiencias y recomendaciones para el Perú. 41 p. IRD Selva - UNALM. FDA, Lima, Perú.

Juárez-Bravo, A., H. Debernardi-De La Vequia, A. Quevedo-Nolasco, F. Malagón-González, y V. Morales-Ramos. 2018. Características físicas del fruto de café (Coffea arabica L.) en Híbridos de Timor. Agroproductividad 11(3):115-120.

López, D. 2010. Efecto de la carga fructífera sobre la roya (Hemileia vastatrix) del café, bajo condiciones microclimáticas del sol y sombra en Turrialba, Costa Rica. Tesis Mag. Sc. Centro Agronómico Tropical de Investigación y Enseñanza (CATIE). 99 p. Turrialba, Costa Rica.

López-García, F.J., E. Escamilla-Prado, A. Zamarripa-Colmenero, y J.G. Cruz-Castillo. 2016. Producción y calidad en variedades de café (Coffea arabica L.) en Veracruz, México. Rev. Fitotec. Mex. 39(3):297-304.

Manchego, R., J. Bustamante, y J. Garnica. 1999. Evaluación agronómica de ocho líneas de café (Coffea arabica) resistentes a roya en la localidad de El Playón, estado Mérida. Bioagro 11(3):97102.

Marín, S., J. Arcila, E. Montoya, y C. Oliveros. 2003. Relación entre el estado de madurez del fruto del café y las características de beneficio, rendimiento y calidad de bebida. CENICAFÉ 54(4):297-315.

Moreno, G., y G. Alvarado. 2000. La variedad Colombia: veinte años de adopción y comportamiento frente a nuevas razas de roya del cafeto. 32 p. CENICAFÉ, Caldas, Colombia.
MINAGRI. 2014. Plan Nacional de Renovaciones de Cafetales. Ministerio de Agricultura y Riego (MINAGRI). Dirección General de Competitividad Agraria, Lima, Perú.

MINAGRI. 2015. Síntesis agroeconómica de café. Ministerio de Agricultura y Riego (MINAGRI). Dirección General de Políticas Agrarias. Dirección de Estudios Económicos e Información Agraria, Lima, Perú.

MINAGRI. 2018. Situación actual del café en el país. Disponible en http://minagri.gob.pe/ portal/485-feria-scaa/10775-el-cafe-peruano (Consulta 01 de marzo de 2018).

Montes, C., O. Armando, y R. Amilcar. 2012. Estudo da infestação e incidência da broca, a ferrugem e a cercosporiose do cultivo do café no departamento do Cauca. Biotecnol. Sector Agropec. Indust. 10(1):98-108.

Montilla, J., J. Arcila, M. Aristizábal, E. Montoya, G. Puerta, C. Oliveros, et al. 2008. Propiedades físicas y factores de conversión del café en el proceso de beneficio. $8 \mathrm{p}$. CENICAFÉ Avances Técnicos 370. Federación Nacional de Cafeteros de Colombia Caldas, Colombia.

Montoya, E. 1999. Caracterización de la infestación del café por la broca y efecto del daño en la calidad de bebida. CENICAFÉ 50(4):245-258.

Pozza, A., H. Martinez, S. Caixeta, A. Cardoso, L. Zambbolim, y E. Pozza, 2001. Influência da nutrição mineral na intensidade da manchade-olho pardo em mudas de cafeeiro. Pesquisa. Agropecuaria. Brasileira 36(1):5360.

Puerta, G. 2000a. Influencia de los granos de café cosechados verdes, en la calidad física y organoléptica de la bebida. CENICAFÉ 51(2):136-150.

Puerta, G. 2000b. Beneficie correctamente su café y conserve la calidad de su bebida. $8 \mathrm{p}$. Avances Técnicos 276. CENICAFÉ, Caldas, Colombia.

Santistevan, E. 2013. Caracterización fenotípica de 33 clones de café Robusta (Coffea canephora) en la comunidad Río Verde, Catón Santa Elena. 86 p. Tesis de Ingeniero Agropecuario. Universidad Estatal Península de Santa Elena, La Libertad, Ecuador.

Severino, L., N. Sakiyama, A. Pereira, G. Mirando, L. Zambolim, y U. Barros. 2001. Caracterização de progênies de café Catimor em Martins Soares, Minas Gerais. Rev. Ceres 48(280):629-648.

Severino, L., N. Sakiyama, A. Pereira, G. Mirando, L. Zambolim, y U. Barros. 2002. Eficiência dos descriptores de cafeeiros (Coffea arabica $\mathrm{L}$ ) na discriminação de linhagens de 'Catimor'. Acta Scientiarum 24(5):1487-1492. 